\title{
COMMENTARY
}

\section{Organ donation and the ethics of muddling through}

Klaus Hoeyer* and Anja MB Jensen

\begin{abstract}
Organ donation offers opportunities for people in critical care units to help save the lives of other patients. It is not always easy, however, to handle the transition from treating a patient to preserving a potential donor, and organ donation consistently provokes ethical questions in critical care units. What do we expect ethics to deliver? In light of a recent ethics conference in Denmark, we suggest that by acknowledging that decisions made in the clinic rarely abide to rational decision trees with clear ethical priorities, we can better learn from each other's experiences. We suggest embracing an 'ethics of muddling through' to enhance relevant reflections and stimulate a productive dialogue among health professionals.
\end{abstract}

\section{A case}

A young girl is rushed into the emergency room following a brutal traffic accident. Serious lesions in the back of the head and lack of pupil reaction and muscular response make it seem pointless for the receiving doctor to commence treatment. The girl is almost certainly dying. Nevertheless, she is intubated and her fluctuant blood pressure is treated. The point is not to restore her to a normal life but to keep her body alive long enough to figure out whether she qualifies as an organ donor. Early identification of potential donors provides better transplant results, the doctor knows, and yet some element of doubt makes intubation of the dying girl difficult. The problem relates to a crucial transition characteristic of modern transplantation medicine as a person shifts positions from a patient-in-need-of-treatment to a donor-in-need-of-conservation.

*Correspondence: klho@sund.ku.dk

Department of Public Health, Faculty of Health Sciences, University of Copenhagen, CSS, Øster Farimagsgade 5, Room 10.0.09, DK-1014 Copenhagen, Denmark

\section{An ethics conference}

Transplantation medicine continually instigates conferences on ethics. The story above was narrated on 9 November 2010, when the Danish Center for Organ Donation (DCO) brought together nurses, scientists, surgeons, and anesthesiologists to discuss the ethical quandaries in transplantation medicine. In particular, intubation and resuscitation of brain dead or near-brain dead patients gave rise to concern, and throughout the day, various cases were discussed in the search for moral guidance. The conference participants took this opportunity for reflection to try to identify key moments of moral decision making and possible principles on which to base such decisions. In fact, the conference participants seemed to proceed as if they were making an evidence-based medical decision: they searched for a decision tree with clear priorities, best evidence (moral principles and specification of the information needed), and steps to follow. But what, really, is the type of decision making at stake in these instances of moral quandary?

\section{Ethics and decision making}

According to classic decision-making theory, a decision is rational when based on full appreciation of available evidence and aimed at defined aims [1]. In as early as 1959, however, Charles Lindblom [2] suggested, in what is now regarded a classic article in organization theory, that good decision making is a 'science of muddling through'. It is never clear what constitutes the full range of evidence, and not least because of the time limits imposed on every critical choice, it is very rare that anyone ever attempts to gather that range of evidence (by whatever standards). Furthermore, decision trees laying out 'the ideal rational decision' tend to neglect unexpected and, in principle, unrelated events. Such unrelated events, however, were often of vital importance in the cases discussed on 9 November and will be familiar to many doctors. Whether or not to resuscitate or intubate a potential donor typically needs to be decided within minutes. If intubated, donors must be kept in intensive care units, where doctors often struggle to find available beds. As a possible consequence, other patients 
are perhaps moved out of intensive care or scheduled operations are cancelled because the only temporary space for a potential donor is the operation theater. These complex decisions must be made at a point in time when it is unclear whether the relatives will consent to the donation or whether the donor medically qualifies. When discussing a case in the conference room, surgeons want to know whether the patient is registered in the national donor registry, but in the emergency room, there is not always time to check this information before a decision must be made. The practicalities of everyday clinical decision making involve a lot of muddling through beyond what decision trees take into consideration.

\section{Muddling through and room for reflection}

The question is, What do we need ethics to do for us? Principled reasoning can be helpful in avoiding systematic prejudice and poorly argued treatment priorities [3]. But the moral problems in clinical decision making in organ donation seem to be more akin to those of organizational decision making in general, and when clinicians deal with them, it is probably useful to turn to a more pragmatic form of bioethics [4,5]. Instead of assuming a moral 'evidence base', we might be of more help to health professionals by acknowledging the lack of universal norms and standard situations. A productive dialogue about the problems people actually handle must appreciate the basic ambiguities surrounding the situations doctors and nurses face as patients become donors [6]. Health professionals dealing with new technologies in critical care units act in many instances as what anthropologist Rayna Rapp [7] calls 'moral pioneers': they need to create norms beyond the guidance of existing ones. When discussing difficult decisions, clinicians must reflect on real-life situations such as those presented at the conference, rather than rest on presumptions about a point of total clarity at which the 'real' ethical decision was made. We contend that the kind of dialogue that health professionals need is better facilitated by an ethics of muddling through - which does not presume clarity where there is none - than by a set of principles that they rarely get the chance to apply. Consequently, we suggest rethinking more generally what we want ethics to do for us in relation to the issues raised through organ donation, in which norms are constantly negotiated and challenged in the messy and complex context of everyday clinical decision making.

\section{Competing interests}

The authors declare that they have no competing interests.

\section{Acknowledgments}

The authors thank the speakers and participants at the meeting in Middelfart, Denmark, on 9 November 2010 and the Danish Center for Organ Donation (DCO) for supporting this commentary. AMBJ thanks the Danish Research

Council for Culture and Communication for funding.

Published: 24 January 2011

\section{References}

1. Simon HA: Models of Man - Social and Rational. New York, NY: John Wiley \& Sons; 1967:vii-3.

2. Lindblom CE: The science of 'muddling through'. Public Administration Review 1959, (19):79-88.

3. Beauchamp TL, Childress JF: Principles of Biomedical Ethics. 5th edition. Oxford, UK: Oxford University Press; 2001.

4. Elliott C: Introduction: treating bioethics. In Slow Cures and Bad Philosophers: Essays on Wittgenstein, Medicine, and Bioethics. Durham, NC: Duke University Press; 2001:1-15.

5. McGee G: Introduction to the second edition. In Pragmatic Bioethics. 2nd edition. Edited by McGee G. Cambridge, MA: The MIT Press; 2003:xi-xvi.

6. Ross LF, Thislethwaite JR Jr.: Shared decision making in deceased-donor transplantation. Lancet 2006, 368:333-337.

7. Rapp R: Moral pioneers: women, men and fetuses on a frontier of reproductive technology. In Embryos, Ethics, and Women's Rights: Exploring the New Reproductive Technologies. Edited by Baruch E, D'Adamo AF, Seager J. New York, NY: Haworth Press; 1988:101-116.

doi:10.1186/cc9379

Cite this article as: Hoeyer K, Jensen AMB: Organ donation and the ethics of muddling through. Critical Care 2011, 15:109. 